\title{
Alléger les primes par un financement uniforme
}

\author{
Jürg Schlup \\ Dr méd., président de la FMH
}

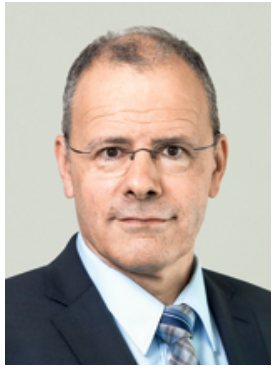

La hausse des primes d'assurance-maladie est un sujet récurrent. Le besoin d'agir dans ce domaine, dont prend conscience le monde politique, prend cependant une nouvelle ampleur comme le montrent les motions déposées récemment au Parlement sur les tarifs étatiques et le budget global. ${ }^{1}$ Ces délibérations menées avec sérieux portant sur le rationnement dans les soins ambulatoires suscitent avant tout l'incompréhension en raison de leur impact négatif sur la prise en charge des patients et l'évolution des coûts. L'inactivité des politiques, qui reportent d'année en année la recherche de solutions pour le principal obstacle au gain d'efficience, à savoir la différence de financement entre prestations ambulatoires et hospitalières, provoque l'agacement. Voici quelques faits importants qui donnent à réfléchir:

\section{Le financement uniforme freine la hausse des} primes provoquée par le transfert des prestations hospitalières vers le secteur ambulatoire.

- Les primes des caisses-maladie ne reflètent pas les coûts de la santé: chaque année, on nous dit que les dépenses de santé augmentent et que, par conséquent, les primes doivent aussi augmenter, mais des détails essentiels sont passés sous silence. En effet, seuls $37 \%^{2}$ des coûts de la santé sont financés par les primes. Alors que les coûts ont augmenté de $72 \%$ depuis 1996 , les primes ont progressé de $107 \%{ }^{3}$; soit nettement plus. Comment est-ce possible?

initiatives parlementaires 17.401 et 17.402; et les réflexions à ce sujet dans Schlup J.Mauvaises propositions de solution pour un problème sérieux, Bull Méd Suisses. 2017;98(9):271.

2 Coûts et financement du système de santé par fournisseur de prestations et par régime de financement. OFS Neuchâtel: 27.10.2016.

3 Système de santé Suisse. Edition 2017. Interpharma: Bâle.

4 PwC. Ambulatoire avant stationnaire-Ou comment économiser un milliard de francs chaque année.http://www.pwc.ch /fr/secteurs-d-activite/ secteur-de-la-sante.html hausses de primes: les traitements ambulatoires sont totalement à la charge des primes, à l'inverse des traitements hospitaliers financés majoritairement par les impôts. Ces subventions fiscales disparaissent lorsque les traitements hospitaliers peuvent, grâce aux progrès de la médecine, être fournis en ambulatoire. Par conséquent, les primes augmentent même si le coût des traitements a été réduit, et la part de dépenses de santé financée par les primes individuelles des caisses-maladie s'accroît.

- Le secteur ambulatoire n'est pas source mais amortisseur de coûts: les soins ambulatoires sont peu onéreux. Qui veut freiner la hausse des coûts de la santé doit développer les soins ambulatoires et non les limiter. Une analyse de $\mathrm{PwC}$ Suisse ${ }^{4}$ a récemment montré que les possibilités non utilisées de traitements ambulatoires représentent un potentiel d'économie annuel d'un milliard de francs. Cela prouve de manière nette qu'il n'est pas rare que la hausse des coûts du secteur ambulatoire soit opposée injustement à des économies proportionnellement plus importantes réalisées à d'autres endroits.

- Les incitatifs erronés de notre système de financement ne permettent pas de faire fructifier les potentiels d'économies: une assurance-maladie qui miserait systématiquement sur «l'ambulatoire avant l'hospitalier», en vérifiant attentivement les garanties de prise en charge des traitements hospitaliers, permettrait aux cantons d'économiser beaucoup d'argent, mais devrait financer seule la hausse de traitements ambulatoires qui en résulterait. Elle devrait par conséquent augmenter les primes et verrait ainsi ses assurés partir à la concurrence. Aujourd'hui, il n'est pas possible de répercuter sur les assurés les économies liées à une réduction des traitements hospitaliers.

Dans notre système de santé, les potentiels d'économies ne sont pas exploités, parce que les avantages ne reviennent pas à celui qui investit, et même les efforts pour un gain d'efficacité se traduisent par de nets inconvénients. En revanche, un financement uniforme des prestations ambulatoires et hospitalières mettrait la priorité sur l'intérêt commun, celui d'atteindre des coûts globaux les plus faibles possible. La transition serait facile si une part constante du financement canto-

\section{Les possibilités non utilisées de traitements} ambulatoires représentent un potentiel d'économie annuel d'un milliard de francs.

nal venait alimenter une instance commune. La compétence cantonale en matière de soins serait maintenue. Avant de parler de tarifs étatiques, budgets globaux et rationnement, il s'agirait d'exploiter enfin ce potentiel d'économie, qui allégerait les primes sans limiter les prises en charge. 